\title{
Numerical analysis using a fixed grid method for cardiovascular flow application
}

\begin{abstract}
Motivated by the current interest in the numerical simulation of biological flows in the human body, we develop a new method to simulate fluid flow embedded in a solid region. The novelty of this method lies on the use of a fixed grid in the entire computational domain. The formulation is an extension of the multiphase fluid flow that belongs to the category of the penalty method, where high viscosity is imposed on a solid region. A free open source library, namely, OpenFOAM, is used to integrate high order and advanced numerical schemes into these computational formulations. The Monotone Upstream System for Conservation Laws (MUSCL) scheme by van Leer, with a harmonic limiter from the category of the total variation bounded (TVB) scheme, is used for cell face interpolation. The robustness and accuracy of the solver are compared with the benchmark test case, namely, the free fall of a solid sphere. The test case validates that the rigidity of the solid sphere is ensured with the selected high viscosity ratio. The accurate terminal velocity of the falling solid sphere proves the no-slip condition at the solid-liquid interface. As a real application implementation, the flow on a simplified idealized model of heart valve stenosis is presented.
\end{abstract}

Keyword: Fluid structure interaction (FSI); Heart valve; Immersed boundary; Openfoam; Volume of fluid (VOF) 